\title{
MÉTODOS GEOESTATÍSTICOS APLICADOS À MODELAGEM GEOMETALÚRGICA
}

\author{
M. VIEIRA*, A. MENDONÇA e J. F. C. L. COSTA \\ Universidade Federal do Rio Grande do Sul \\ vieiracas@yahoo.com.br*
}

Artigo submetido em novembro/2015 e aceito em dezembro/2015

DOI: $10.15628 /$ holos.2015.3727

\begin{abstract}
RESUMO
Diversos fatores interferem na eficiência do processamento mineral e seu correto entendimento aprimora a performance operacional das plantas de beneficiamento. A geometalurgia tem se tornado uma ferramenta importante nesse cenário, pois o conhecimento das propriedades da rocha e das variáveis de processo possibilita uma análise econômica mais acurada do processo, melhoria no prognóstico do planejamento de lavra e na avaliação dos riscos do projeto. Nesse estudo, a modelagem por métodos geoestatísticos das variáveis metalúrgicas e de processo de uma jazida de zinco é aplicada para aumentar a previsibilidade do material enviado para o beneficiamento e para o melhor direcionamento das rotas de processo. A jazida localiza-se no mais importante distrito zincífero do Brasil, no noroeste de Minas Gerais.
\end{abstract}

A metodologia utilizada para a modelagem consistiu em definir, inicialmente, os parâmetros de relevância para o projeto e dividi-los em categorias de acordo com a similitude e conectividade espacial. Assim sendo, o depósito foi classificado em quatro tipologias, a partir das quais foram elaborados modelos multivariáveis por meio de krigagem de indicadores e de simulação sequencial de indicadores. $\mathrm{O}$ resultado obtido possibilitou a estimativa da variabilidade do minério e o conhecimento do impacto causado por essa variabilidade na recuperação do metal. O modelo geometalúrgico das tipologias auxilia no prognóstico da recuperação metalúrgica de cada unidade seletiva de lavra. Ainda, serve como valiosa ferramenta para tomadas de decisões, no planejamento de lavra e para melhor definição das rotas de processo referentes ao tratamento do material lavrado.

PALAVRAS-CHAVE: Geometalurgia, modelagem, krigagem de indicadores, simulação sequencial de indicadores.

\section{GEOSTATISTICAL METHODS APPLIED TO GEOMETALLURGICAL MODELING}

\begin{abstract}
Several factors affect a mineral processing and their knowledge improves the operating performance of processing plants. Geometallurgy has become an important tool in this context, for knowing rock properties and process variables enable an accurate economic examination of the process, improvement in the forecast of mining planning and assessment of project risks. In this study, geostatistical modeling of metallurgical and process variables is applied to increase the predictability of material processing and for better planning of the process routes. The aim of this reseach is to evaluate the typological position uncertainty of a zinc deposit of northwest Minas Gerais by geostatistical techniques and the impact generated by each typology
\end{abstract}

behavior in mining and ore processing. The geostatistical techniques consist in defining the major parameters of the project and dividing them into categories according to their spatial similitude and connectivity. Therefore, the deposit was classified into four typologies, from which were developed multivariate models by indicator kriging and sequential indicator simulation. The result enabled to estimate the variability of the ore and the knowledge of the impact of this variability in the metal recovery. The metallurgical model assists in predicting metallurgical recovery of each selective mining unit. Also is a valuable tool for decision making, for future mining planning and better definition of process routes concerning to ore treatment.

KEYWORDS: Geometallurgy, modeling, indicator kriging, sequential indicator simulation. 


\section{INTRODUÇÃO}

A geometalurgia é uma atividade interdisciplinar que estuda a relação de causa e efeito entre as variáveis geológicas e metalúrgicas relevantes em todas as etapas de produção e planejamento mineiro (Beniscelli, 2011; Baumgartner et al., 2011; Lamberg, 2011), e que objetiva agregar valor econômico à extração de recursos naturais, fornecendo informações relevantes e conhecimento sobre as variáveis metalúrgicas de um depósito, representadas por modelos espaciais. A geometalurgia surgiu da necessidade de se ter um melhor aproveitamento do corpo de minério e controle sobre os riscos inerentes à mineração (Lamberg, 2011). A modelagem geometalúrgica permite antever problemas nas etapas posteriores de mineração e tratamento de minérios (Motta, 2014), contribuindo para um melhor planejamento, minimizando riscos do processamento e otimizando os planos de produção nas plantas de beneficiamento.

A base para uma boa modelagem é a atribuição de dados metalúrgicos para um volume específico de um depósito mineral, em função do conhecimento adquirido através da sua caracterização geológica, mineralógica, química e física, suportada por critérios de representatividades adequados (Beniscelli, 2011). Além disso, é necessário que haja uma integração entre geólogos, geoestatísticos, metalúrgicos e planejadores de mina, de forma que se maximize o valor econômico do recurso (Stradling, 2011; Beniscelli, 2011).

Atualmente, o termo "geometalurgia" deixou de referir-se apenas à caracterização mineralógica do mineral de interesse e de ganga e passou a ter um conceito mais amplo. A geometalurgia moderna abrange um conceito de sustentabilidade geometalúrgica que consiste na interpretação, análise, avaliação e validação de todos os aspectos técnicos que suportam os resultados de cada uma das atividades associadas ao projeto, de acordo com o avanço na obtenção de dados técnicos e econômicos, para converter recursos minerais em reservas de minério. Assim sendo, a caracterização mineralógica e metalúrgica é aplicada em projetos conceituais, de préviabilidade dos recursos e em projetos finais de viabilidade econômica, maximizando seus valores (Dobby et al., 2004, apud Motta, 2014; Baumgartner, 2011).

A utilização dos parâmetros geometalúrgicos na modelagem do depósito, tais como variação da dureza, mineralogia, grau de liberação e porosidade, possibilita a identificação prévia da variabilidade do comportamento metalúrgico de diferentes tipologias e, ainda, dá apoio à estabilidade dos processos. Essa caracterização tecnológica, que não passa de uma visão restrita da geometalurgia, identifica os diferentes desempenhos do minério no beneficiamento de minério (Lemos, 2013).

Nesse trabalho, estudam-se as tipologias que interferem no processamento de uma mina de zinco silicatado, cujos principais minerais minério são willemita $\left(\mathrm{Zn}_{2} \mathrm{SiO}_{4}\right)$ e hemimorfita $\left(\mathrm{Zn}_{4} \mathrm{Si}_{3} \mathrm{O}(\mathrm{OH})_{2}\right)$.

No depósito em questão, a presença de hematita e argilominerais no minério de zinco gera finos (lama), ocasionando o efeito slime coating, que consiste no recobrimento das partículas maiores pelos finos. Esses minerais acarretam, ainda, baixa liberação do mineral minério. Quando há uma quantidade elevada de sulfetos em lentes associadas ao minério willemítico, a interação entre as partículas de silicato de zinco e reagentes de flotação fica comprometida, prejudicando a recuperação do zinco a partir da willemita. Já o minério rico em dolomita também gera o efeito 
slime coating, devido à presença de finos gerados, causando aumento no consumo de reagentes da flotação. Além disso, ocorrem perdas durante a lixiviação ácida, elevando o consumo de ácido e produzindo solução com altos teores de magnésio, desfavorecendo a eletrólise de zinco (Lemos, 2011).

Da necessidade de otimização das rotas operacionais de uma usina de beneficiamento, do prognóstico da performance do processo e do aperfeiçoamento do planejamento, surgiu o interesse em compreender e modelar a distribuição das variáveis litológicas que interferem nesses processos. A modelagem a partir de informações tipológicas que afetam diretamente as etapas de beneficiamento e metalurgia tem por objetivo o aumento da recuperação, correlacionando os parâmetros de tratamento de minério às características geológicas.

\section{MATERIAL E MÉTODOS}

Foram realizados 140 furos de sondagem em uma malha irregular, com espaçamento entre os furos variando de 20 a 30 metros e entre as amostras de um mesmo furo (down the hole), de 0,4 a 30 metros. As amostras foram compositadas em um suporte de 2,5 metros, gerando 9.750 amostras.

A modelagem foi realizada classificando o depósito em quatro tipologias, de acordo as diferentes respostas e necessidades apresentadas no processamento:

- brecha willemítica (BXW): brecha hidrotermal e/ou tectônica, correspondendo à tipologia mais rica em zinco;

- brecha dolomítica (BXD): brecha hidrotermal e/ou tectônica, possui zinco, porém contém alto teor de dolomita e, por conseguinte, de magnésio, o que prejudica o beneficiamento do zinco. Quando apresenta alto teor de zinco, pode ser considerada minério;

- brecha hematítica (BXH): tipologia com alto teor de ferro, além de possuir zinco; e

- estéril: diversos tipos de rocha que não possuem zinco em sua composição.

Para proceder à estimativa das classes tipológicas em todo o depósito, os dados originais foram transformados em indicadores, os quais receberam valor 1 para cada tipologia individualmente e zero, caso contrário, conforme a Equação (1):

$$
\left\{\begin{array}{l}
i\left(u_{\alpha} ; s_{l}\right)=1 \text { para } s\left(u_{\alpha}\right)=s_{l} \\
i\left(u_{\alpha} ; s_{l}\right)=0 \text { caso contrário }
\end{array}\right.
$$

em que $i\left(u_{\alpha} ; s_{l}\right)$ é o indicador da categoria $s_{l}$ no local $u_{\alpha}$.

A Figura 1 mostra a proporção de cada classe tipológica no depósito. Observa-se que há um predomínio da brecha dolomítica dentre os tipos de brecha. 


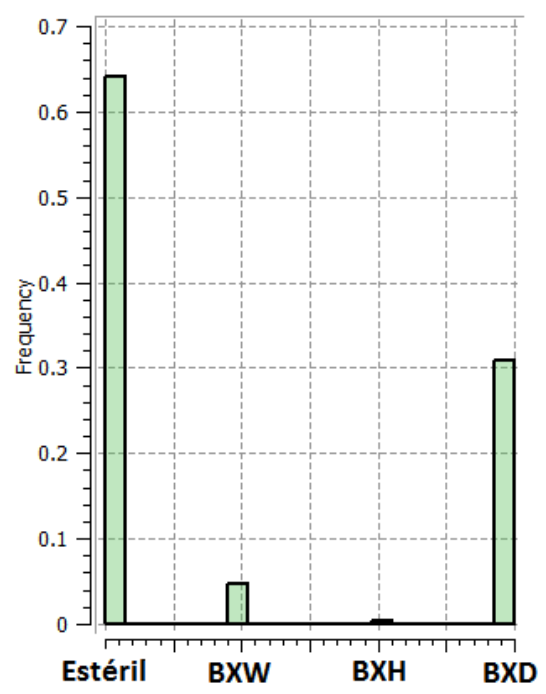

Figura 1: Histograma das tipologias do depósito

Para a estimativa das tipologias da mina, foram utilizadas krigagem ordinária e simulação de indicadores. Estes métodos foram escolhidos para a estimativa por serem categóricas as variáveis estudadas.

Na krigagem de indicadores, as inferências dos valores são obtidas a partir da construção da função de distribuição acumulada, condicionada aos dados amostrados de cada variável. É um método de estimativa flexível, devido à codificação binária dos valores, tendo a vantagem de fornecer precisão local.

A simulação sequencial de indicadores produz possíveis cenários do depósito mineral, os quais são chamados de realizações. 0 método fornece precisão global, com um campo simulado semelhante às estatísticas do campo verdadeiro e permite a avaliação da incerteza associada à estimativa (Goovaerts, 1997). Ambos os métodos permitem a modelagem dos diferentes padrões de continuidade de diferentes intervalos da distribuição.

Para realizar a krigagem de indicadores e a simulação sequencial de indicadores, foi necessário modelar o semivariograma dos quatro indicadores - para modelar o indicador do BXW, utilizou-se correlograma, diferente dos demais.

Todos os indicadores mostraram maior continuidade no azimute de 45 ․

Foi utilizado o software SGeMS (software desenvolvido na Universidade de Stanford, que implementa vários algoritmos de geoestatística) para estimativa das tipologias.

\section{RESULTADOS E DISCUSSÃO}

Duas estimativas foram realizadas: uma em um grid que engloba toda a área e outra em um grid mais delgado, restrito apenas a uma zona de interesse mais mineralizada, a fim de se obter um melhor detalhamento e entendimento do comportamento do minério.

A krigagem dos indicadores reproduziu quatro estimativas, fornecendo, assim, a probabilidade de ocorrência de cada uma das classes tipológicas individualmente. Para a construção de um único mapa que contivesse as quatro tipologias, considerou-se a tipologia com maior probabilidade de ocorrência em cada ponto do grid. 
Como a simulação sequencial dos indicadores gera modelos com todas as tipologias, não foi necessário nenhum procedimento após os resultados iniciais. Um pós-processamento dos dados geraria estimativas individualizadas para as quatro classes tipológicas, que não é o objetivo deste trabalho.

Por ocorrer em proporção mínima (menor que as outras três), o BXH foi subestimado nas estimativas, a ponto de não ser possível detectá-lo. Essa subestimativa é devida à suavização gerada pela krigagem que reduz a proporção das classes nos extremos (baixos e altos).

As Figuras 2 e 3 mostram a krigagem e a simulação sequencial dos indicadores realizadas com todas as tipologias (mapas da esquerda) e detalhando a zona mineralizada (mapas da direita).

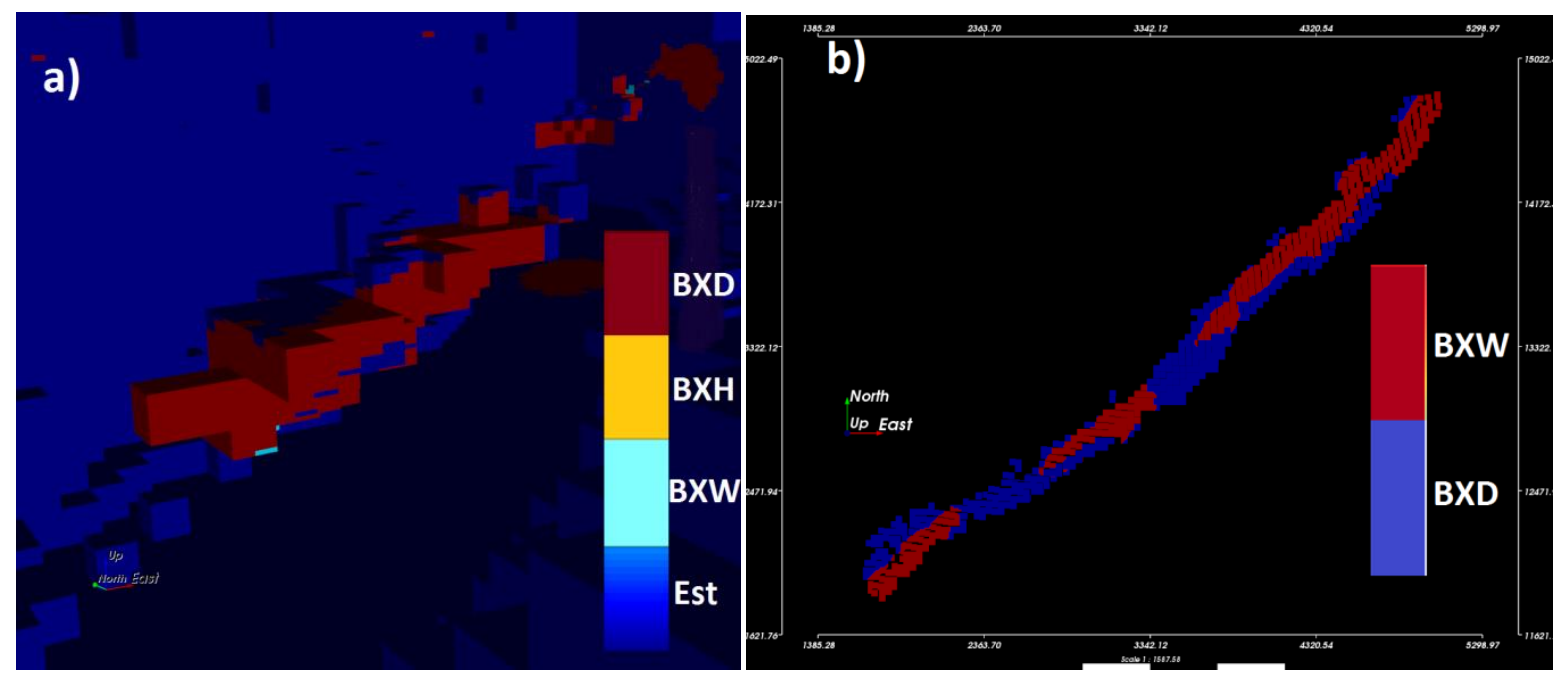

Figura 2: Krigagem dos indicadores em a) grid englobando toda a área e b) detalhe da área mineralizada

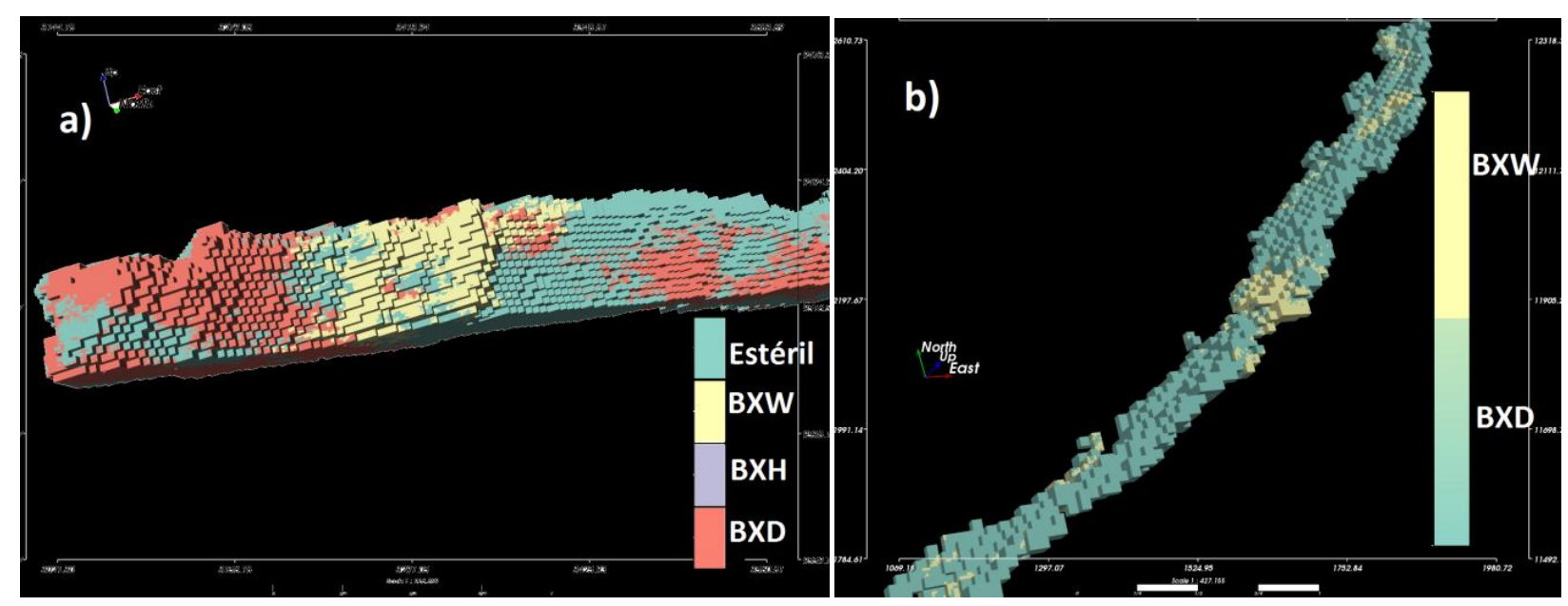

Figura 3: Simulação sequencial de indicadores em a) grid englobando toda a área e b) detalhe da área mineralizada

\subsection{Discussão}

A krigagem dos indicadores forneceu a probabilidade de ocorrência de cada classe tipológica, além de apresentar uma melhor delimitação da zona mineralizada. 
A simulação mostrou vários cenários para a distribuição das tipologias ao longo da jazida. A classe referente ao BXW apresentou frequentes flutuações nos histogramas das realizações, devido à baixa proporção deste no depósito quando comparado com as outras tipologias. A classe referente ao BXH praticamente tornou-se inexistente, devido a sua baixa proporção e consequente suavização gerada pela krigagem.

A estimativa realizada apenas na área de interesse diminuiu a incerteza dos resultados devido ao detalhamento da mesma, além de fornecer uma localização mais acurada do minério.

O conhecimento correto da localização das principais tipologias da mina permite prever o comportamento do minério ao longo de toda a cadeia produtiva, favorecendo desde o planejamento de lavra, passando pelo beneficiamento do minério até a metalurgia.

\section{CONCLUSÕES}

O modelo geometalúrgico do depósito, presente nesse trabalho, fornece um melhor entendimento da distribuição espacial das tipologias, permitindo a determinação da variabilidade do minério. Este modelo contribui para o prognóstico da recuperação metalúrgica de zinco, para a definição das rotas de processo, para o planejamento de mina e para a elaboração do modelo econômico do depósito. Constatou-se que o simples conhecimento do teor do minério não é suficiente para as etapas de planejamento, beneficiamento e metalurgia, sendo necessário conhecer também a tipologia, uma vez que cada uma tem uma particularidade que responde de maneira diferente no processamento mineral. Com a modelagem das tipologias, diminui-se o impacto causado pelas diferenças na qualidade do material a ser processado.

\section{AGRADECIMENTOS}

Os autores agradecem à Votorantim Metais pelos dados concedidos, em especial à geóloga Mariana Gazire, e ao colega do Laboratório de Pesquisa Mineral e Planejamento Mineiro Rafael Caixeta, pelas valiosas contribuições.

\section{REFERÊNCIAS BIBLIOGRÁFICAS}

1. BENISCELLI, J. Geometallurgy - Fifeen Years of Developments in Codelco: Pedro Carrasco Contributions. In: The First AUSIMM International Geometallurgy Conference; 5-7 September. Brisbane, QLD, AUS. 2011.

2. BAUMGARTNER, R. et al. Building a geometallurgical model for early-stage project development - a case study from the Canahuire epithermal Au-cu-Ag deposit, Southern Peru. In: The First AUSIMM International Geometallurgy Conference; 5-7 September. Brisbane, QLD, Australia, 2011.

3. DOBBY, G., BENNETT C., BULLED, D. \& KOSICK, G. Geometallurgical modeling - The new approach to plant design and production forecasting/planning and mining/mill optimization. In: Proceedings 36th Annual Meeting of the Canadian Mineral Processors Conference. Otawa, 2004, pp. 227-240.

4. LAMBERG, P. Particles - The bridge between geology and metallurgy. Center of advanced 
mining and metallurgy (CAMM) - Lulea university of technology, mineral processing. Lulea, Sweden. 2011.

5. LEMOS, M.G. Caracterização geológica e tecnológica do minério de zinco do extremo norte da mina de Vazante, Minas Gerais, MG. Campinas, 2011. Dissertação de mestrado - Instituto de Geociências - Universidade Estadual de Campinas, 2011.

6. LEMOS, M.G. et al. Aplicação da Geometalurgia nos Processos Produtivos Votorantim Metais. Relatório interno Votorantim Metais, Tecnologia Zinco, Unidade Vazante. 2013.

7. GOOVAERTS, P. Geostatistics for Natural Resources Evaluation. New York: Oxford University Press; 1997. 483 p.

8. MOTTA, E.G. Definição de domínios mineralógicos de minério de ferro utilizando krigagem de indicadores. Porto Alegre, 2014. Dissertação de mestrado - Universidade Federal do Rio Grande do Sul, 2014.

9. STRADLING, A. Geometallurgical modeling - A mineral processor's perspective. In: The First AUSIMM International Geometallurgy Conference; 5-7 September. Brisbane, QLD, Australia, 2011.

10. Stanford University, School of Earths Sciences [homepage on the internet]. [cited 2015 May 11] Available from: http://geostat.stanford.edu/research.php?rg_id=53\&_rgpr_id=59. 